\section{Utilization of Saccharose and Maltose by Yeasts}

Work on the utilization of saccharose by Saccharomyces oleaginosus $^{1}$, and other species, has been continued on other yeasts lacking invertase. The results with $S$. rouxii were: $(a)$ negative assimilation in yeast nitrogen base Wickerham (YNB) with 0.5 per cent saccharose, and positive assimilation in the same medium with a 2 per cent sugar concentration; in the latter case, the assimilation is appreciably quicker in Erlenmeyer flasks; (b) slow fermentation in a 2 per cent concentration in yeast infusion (YI), and slightly more active fermentation in yeast autolysate (YA). With $S$. italicus VB 20 the assimilation of saccharose in YNB was also negative with 0.5 per cent saccharose, and positive with 2 per cent; the fermentation was extremely weak in YI and YA.

Further, a series of yeasts was chosen capable of assimilating saccharose, but not raffinose, and it was proved that they lacked beta-fructosidase; their behaviour in the presence of saccharose varied from extremely active fermentation (C. tropicalis) to very weak (H. angusta $A c 455, C$. lusitanae $A c 475$ ) to a negative fermentation (C. pulcherrima, $T$. ernobii, $H$. californica, and the majority of the $C$. lusitanae strains).

A similar behaviour with maltose was found only in the yeasts lacking invertase, $S$. rouxii $M e 3$, that fermented maltose (slowly in YI and more rapidly in YA) and did not assimilate it in YNB with 0.5 per cent sugar; in the same manner, two invertase-positive yeasts $(C$. utilis Ac 551 and S. kluyveri Ac 316) fermented maltose in YA, but not in YI.

Considering the general problem of utilizing saccharose and maltose, we note that the following points stand out, at least in the group of yeasts treated: $(a)$ Whenever a yeast ferments maltose, it ferments saccharose, even though this fermentation be weak or may need special media. (b) A yeast may ferment saccharose in the absence of invertase, but can neither assimilate nor ferment maltose (S. wickerhamii), or can only assimilate it $(H$. angusta $A c 455$ ). (c) There are yeasts that ferment glucose and assimilate maltose and/or saccharose, but are incapable of fermenting them. (d) Within one species, there may be appreciable variation between different strains with regard to the assimilation or fermentation of maltose and/or saccharose (S. rouxi $i, S$. kluyveri, $S$. italicus, $C$. lusitanae, $C$. utilis).

From the quantitative point of view, for example the amount of sugar utilized, there are differences between cultures. For example, with S. oleaginosus, S. hienipiensis, $S$. italicus, C. tropicalis, some strains of $C$. lusitanae, and others, the maltose utilized in 10 days in YNB represents practically 100 per cent of the initial amount ( 2 per cent), while $S$. rouxii, S. lactis, $C$. utilis var. major, other strains of $C$. lusitanae, and others, the corresponding figure after 20 days is only 10 to 20 per cent. The culture medium. used has a profound influence on the rate of sugar utilization. The same happens with saccharose: C. tropicalis Ac 433 utilizes 100 per cent of the initial amount (4 per cent) in $48 \mathrm{~h}$, while C. lusitanae $A c 19$ utilizes 17 per cent, $T$. ernobii 8 per cent, and $H$. californica 10 per cent. The addition of various peptones to the growth medium has no influence on the results. In the case of saccharose, YA may not be substituted by YNB plus vitamin-free acid hydrolysed casein, using $S$. oleaginosus, $S$. italicus $V B 20, S$. hienipiensis, $S$. rouxii, $H$. angusta. On the other hand, $S$. rouxii $M e$, which does not assimilate maltose in YNB, will do so if it is complemented by 'Bacto' vitamin-free casamino-acids. In small inoculations, adaptation to maltose or saccharose has no influence on utilization of the saccharose by $S$. oleaginosus, S. hienipiensis, $S$. italicus VB $20, S$. rouxii, or by maltose by $S$. rouxii $M e$, unlike that which occurs with other inducible enzymes.
We see, therefore, that with regard to the utilization of saccharose and/or maltose by yeasts lacking invertase, it is possible to use them in simple media (YNB) or more complicated ones (YA) which may be fermentative or merely assimilative. We also observe that if there is invertase, the saccharose is fermented, and equally, if there is melibiase, the melibiose is fermented; on the other hand, maltose and saccharose, in the absence of invertase, may be assimilated and not fermented: a similar case occurs with beta-galactosidase in S. marxianus. This seems to coincide with the fact that beta-fructosidase (invertase) and alpha-galactosidase (melibiase) involve external hydrolysis.

From these results we may deduce that: (i) it is probable that utilization of saccharose by yeasts lacking invertase may be due, at least in some cases, to a different enzymatic system from that used in the utilization of maltose; (ii) in the utilization of saccharose and maltose diverse factors (such as medium, concentration of sugar, aerobiosis, strain of yeast) have such a decisive influence that the sugar utilization pattern is unstable; this must be taken into account from the taxonomic point of view; (iii) the utilization of saccharose in the absence of invertase without external hydrolysis having been confirmed, it seems logical to admit the existence of a similar system to that of maltozimase ${ }^{2}$, but the fact that yeasts that ferment glucose are only capable of assimilating maltos $\theta^{8}$ or saccharose remains unexplained.

\section{J. Santa María}

Sección de Bioquímica,

Instituto Nacional de Investigaciones Agronómicas, Madrid 3.

1 Santa María, J., Nature, 195, 1201 (1962).

2 Robertson, J. J., and Halvorson, H. O., J. Bacteriol., 73, 195 (1957).

${ }^{3}$ Roberts, C., Winge, Ø., and Wynants, J., C.R. Lab. Carlsberg, 32, 305 (1961).

\section{Action of Phospholipase $\boldsymbol{C}$ on Influenza Virus}

INFLUENZA virus is known to contain large amounts of phospholipid. By treating the virus with phospholipase $C$, considerable amounts of acid-soluble phosphoric compounds are released, and the virus becomes sensitive to the action of proteinase from Streptomyces griseus (proteinase SG, ref. 1) with reduction of its infectivity.

The 'A2/Kumamoto $Y / 5 / 57$ ' strain $^{2}$ of influenza virus (non-avid) was used for this experiment. Virus was labelled with ${ }^{32} \mathrm{P}$ by the method of Liu et $a .^{3}{ }^{3}$, and was purified by three cycles of adsorption-elution from chicken red colls, followed by high- and low-speed centrifugation. The final product was suspended in veronal buffered saline, $p \mathbf{H} 7 \cdot 8$, and tested for contamination with non-viral ${ }^{32} \mathrm{P}$ by determining the Geiger count before and after adsorption with red cells. The preparations had an average hrmagglutinin titre of 10,240 and an average Geiger count of 5,480 c.p.m./ml. Of this radioactivity more than 98.5 per cent was carried by the virus particles, and was adsorbable on the red cells. Attempts were made to determine the relative distribution of the isotope in the virus phospholipid. Labelled virus was fractionated by a modification of the Schmidt-Thannhauser technique ${ }^{4}$. The cold trichloroacetic acid (TCA), hot alcohol-ether, hot TCA-soluble fractions, and the residue, were assayed for radioactivity. On average, 15, 50, 24 and 11 per cent of the label were found respectively in cold TCA, hot alcohol-ther, hot TCA fractions and residue. Phospholipase $C$, kindly supplied to us by Dr. H. Ikezawa of this Institute, had been purified from $C l$. perfringens type $A \alpha$-toxin by the ammonium sulphate and alcohol precipitation procedure ${ }^{5}$. Streptomyces griseus proteinase, which is known to have extremely broad substrate specificity, was obtained from the Institute of Physical and Chemical Research, Tokyo.

Aliquots of labelled virus were treated with (1) phospholipase $C$, varying the concentration of enzyme and 